HNO 2009.57:300-301

DOI 10.1007/s00106-009-1890-7

Online publiziert: 26. März 2009

(c) Springer Medizin Verlag 2009

\section{W. Heppt}

Klinik für Hals-, Nasen-, Ohrenkrankheiten, Kopf-, Hals-, Plastische Gesichtschirurgie, Städtisches Klinikum Karlsruhe gGmbH, Karlsruhe

\title{
Hauttumoren des Gesichts
}

\author{
Eine Herausforderung für Hautärzte \\ und Hals-Nasen-Ohren-Ärzte
}

Hauttumoren weisen in den letzten Jahrzehnten weltweit eine besorgniserregende Zunahme auf. Die höchsten Zuwachsraten findet man auf dem australischen Kontinent. Hauptverantwortlich für diese Entwicklung ist der Anstieg der kumulativen UV-Licht-Dosis durch Abbau der schützenden Ozonschicht. Neben dem natürlichen Sonnenlicht tragen hierzu auch künstliche UV-Quellen wie Sonnenbank, Photolichttherapie oder hochfraktionierte ionisierende Behandlung bei. Die Zunahme effektiver immunsuppressiver Therapien ist ein weiterer begünstigender Faktor.

\section{( Basaliome und aktinische Keratosen stellen die häufigsten Gesichtstumoren dar}

Basaliome und aktinische Keratosen stellen die häufigsten Gesichtstumoren dar, eine Vielzahl gutartiger und bösartiger Tumoren sind davon differenzialdiagnostisch abzugrenzen. Im Artikel von Markus Braun-Falco findet sich eine übersichtliche Zusammenstellung, die ihr Credo auf die "saubere" histopathologische Diagnosestellung legt. Es wird betont, dass ohne klare Zuordnung der Tumorentität keine adäquate Therapie und Verlaufskontrolle möglich ist. Diese Aussage erscheint auf den ersten Blick trivial, gewinnt aber gerade bei offenkundig eindeutigen Befunden tiefere Bedeutung. Ein im Frühstadium als aktinische Keratose fehldiagnostiziertes kutanes Lymphom wird nicht nur übersehen, sondern auch fälschlicherweise einer Laserablation zugeleitet, was die Diagnostik im weiteren Verlauf verwischt. Fatal ist das Übersehen eines malignen Melanoms im Frühstadium unter der Fehldiagnose Verruca vulgaris. Die Entscheidung für die im Einzelfall richtige chirurgische Resektion, Laserablation, Bestrahlung oder Chemotherapie ist vor Einleitung der Therapie histopathologisch zu treffen.

Die chirurgische Therapie liefert bei den meisten bösartigen Tumoren die höchsten Heilungs- und niedrigsten Rezidivraten. Dies gilt v. a. für die große Zahl der Basaliome. Mit einem Lebenszeitrisiko von $30 \%$ und einer Inzidenz von 100/100.000 Einwohner ist das Basalzellkarzinom in Deutschland der häufigste nicht benigne Hauttumor. Die Tatsache, dass sich die Inzidenz in den letzten 10 Jahren verdoppelt hat, verdeutlicht die dramatische epidemiologische Entwicklung. Da Basaliome überwiegend an sonnenexponierten Regionen im Gesicht, v. a. an Nase, Stirn, Wange und Ohrmuschel vorkommen, ist neben dem Hauttarzt initial häufig der Hals-Nasen-OhrenArzt gefordert. Sind Basaliome durch kleine Stanzbiopsien gesichert, werden sie mit einem Sicherheitsabstand von 3-5 $\mathrm{mm}$ reseziert. Bei Spinaliomen, malignen Melanomen oder Merkelzell-Tumoren sind die Resektionsgrenzen, sofern anatomisch möglich, weiter zu ziehen. Nach den aktuellen onkologischen Leitlinien der Deutschen Dermatologischen Gesellschaft aus dem Jahr 2008 (http://www.jddg.de) betragen sie abhängig vom Tumortyp und von Parametern wie der Größe, der vertikalen Tumordicke und dem Vorliegen einer Ulzeration bis $\mathrm{zu} 2 \mathrm{~cm}$.
Die Deckung der entstehenden Defekte orientiert sich an den Prinzipien der ästhetischen Gesichtschirurgie und erfordert sowohl Erfahrung als auch Geschick. Der Autor unterstreicht die herausragende Bedeutung einer individuellen Planung, die auf der Wahl der richtigen Operationstechnik aus einer Vielzahl von Möglichkeiten beruht.

\section{( Die chirurgische Therapie liefert bei den meisten bösartigen Tumoren die höchsten Heilungs- und niedrigsten Rezidivraten}

Die klassische chirurgische Resektion wird zwar der Vielzahl von Patienten mit lokalisierten Tumoren gerecht, stößt bei der in der Zukunft weiter zunehmenden Problematik der Feldkanzerisierung jedoch an ihre Grenzen. Auf diese Thematik geht Eckart Haneke in seinem Referat ein. Er gibt eine Übersicht über die heute verfügbaren Möglichkeiten der nichtchirurgischen Behandlung von Präkanzerosen und frühinvasiven Hautkarzinomen. Klar strukturiert gibt er eine differenzierte Beurteilung für die Therapieoptionen lokale Zytostase, photodynamische Therapie und lokale Immunmodulation und beleuchtet auch die intra- und peritumoröse Injektion von Zytostatika wie die Anwendung von Interferon und neuer krebsspezifischer Mittel.

Die Darstellung stellt zukunftsorientierte Therapiemodalitäten vor, ohne die kritische Distanz zu verlieren. So vertritt Haneke trotz der guten Erfolge in der Behandlung der aktinischen Keratosen die 
Meinung, dass Basalzellkarzinome nur in bestimmten Fällen eine Indikation für eine photodynamische Therapie darstellen. Derartige kritische, evidenzbasierte Einschätzungen neuer Behandlungsverfahren sind heute mehr denn je gefordert, in einer Zeit, in der sich Patienten bei der Wahl der Therapie mehr und mehr von Internetempfehlungen leiten lassen.<smiles>CCC1(C)CCCC1C(C)C</smiles>

\section{Korrespondenzadresse} Prof. Dr. W. Heppt

Klinik für Hals-, Nasen-, Ohren-
krankheiten, Kopf-, Hals-,
Plastische Gesichtschirurgie,
Städtisches Klinikum
Karlsruhe gGmbH
Moltkestraße 90,
76133 Karlsruhe
annette.taschins-
ky@klinikum-karlsruhe.de

Interessenkonflikt. Der korrespondierende Autor gibt an, dass kein Interessenkonflikt besteht.
Liebe Leserinnen, liebe Leser,

${ }_{\text {„HNO }} \mathrm{HN}$ bietet Ihnen jeden Monat umfassende und aktuelle Beiträge zu interessanten Themenschwerpunkten aus allen Gebieten der HNO.

Möchten Sie ein bereits erschienenes Heft nachbestellen? Die folgenden Ausgaben können Sie direkt bei unserem Kundenservice zum Preis von je EUR 32,- beziehen:

\section{9}

- 1/2009 Hörscreening

- 2/2009 Preisträger

- 3/2009 Implantierbare Hörsysteme

- 4/2009 Gesichtschirurgie

- 5/2009 Kongressheft

- 6/2009 Cochlear Implant

- 7/2009 Nebenhöhlenerkrankungen

- 8/2009 Lebensqualität

- 9/2009 Computerassistierte Chirurgie

- 10/2009 Plastische Operationen

- 11/2009 Orbita

- 12/2009 Begutachtung

(Änderungen vorbehalten)
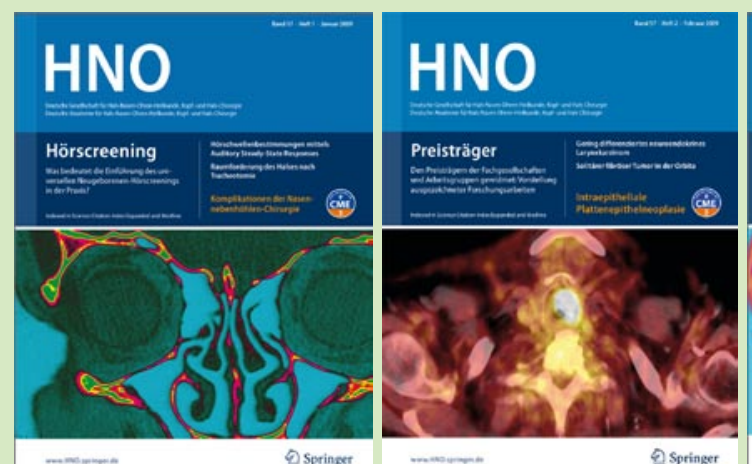

\section{HNO}
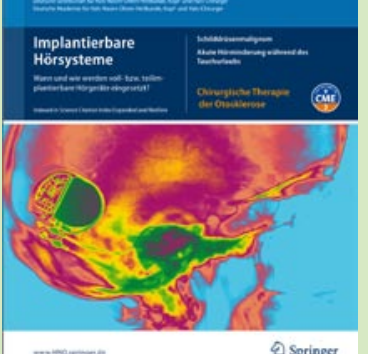

So erreichen Sie unseren Kundenservice:

Springer-Verlag

Kundenservice Zeitschriften

Haberstraße 7

69126 Heidelberg

Tel.: +49 $6221345-4303$

Fax: +49 $6221345-4229$

E-Mail: subscriptions@springer.com

www.HNO.springer.de 